\title{
ROYDEN'S MAP BETWEEN RIEMANN SURFACES ${ }^{1}$
}

\author{
BY MITSURU NAKAI
}

Communicated by A. E. Taylor, June 10, 1966

Let $R$ and $R_{j}(j=1,2)$ be Riemann surfaces, either open or closed. We denote by $M(R)$ Royden's algebra associated with $R$, and by $R^{*}$ Royden's compactification of $R$ (see [5], [6], and [7]). We have seen in [5] that every algebraic isomorphism of $M\left(R_{1}\right)$ onto $M\left(R_{2}\right)$ induces (and is induced by) a quasiconformal mapping of $R_{1}$ onto $R_{2}$. In other words, the algebraic structure of $M(R)$ characterizes the quasiconformal structure of $R$. In this connection there naturally arises the following question: What can we say about the topological structure of $R^{*}$ ? This question leads us to a new notion, Royden's map, which seems to be of considerable function-theoretic interest.

Here we report, without proofs, some of the properties of Royden's maps. Details will be published elsewhere.

1. Moduli of $A$-sets. An open subset $G$ of $R$ is called normal if for any point $z$ in $\partial G$ there exists a parametric disk $U$, with center $z$, such that $\partial G \cap U$ is a simple arc connecting two boundary points of $U$.

An $A$-set $A$ is a pair $\left(G_{1}, G_{2}\right)$ of two nonempty normal open sets $G_{1}$ and $G_{2}$ in $R$ with $G_{1} \supset \bar{G}_{2}$. An annulus in a parametric disk is an example of an $A$-set.

We associate with an $A$-set $A=\left(G_{1}, G_{2}\right)$ a family $\{\phi\}$ of functions $\phi$ which are continuous on $\bar{G}_{1}-G_{2}$, of class $C^{1}$ in $G_{1}-\bar{G}_{2}$, and have boundary values $\phi \mid \partial G_{j}=j(j=1,2)$. The modulus of $A$, denoted $\bmod A$, is the number in $[0, \infty)$ given by

$$
\bmod A=2 \pi / \inf _{\phi \in\{\phi\}} D(\phi),
$$

where $D(\phi)$ is the Dirichlet integral of $\phi$ taken over $G_{1}-\bar{G}_{2}$. If $A$ is an annulus in a parametric disk, then this definition coincides with the usual one.

2. Royden's map. A topological mapping $T$ of $R_{1}$ onto $R_{2}$ carries an $A$-set $A=\left(G_{1}, G_{2}\right)$ on $R_{1}$ to the $A$-set $T A=\left(T G_{1}, T G_{2}\right)$ on $R_{2}$. We call $T$ a Royden's map if there exists a constant $K(A) \geqq 1$ such that

$$
K(A)^{-1} \bmod A \leqq \bmod T A \leqq K(A) \bmod A,
$$

for every $A$-set $A$ on $R_{1}$. Here $K(A)$ may depend on $A$. If we can find

1 The work was sponsored by the U. S. Army Research Office-Durham, Grant DA-AROD-31-124-G 742, University of California. Los Angeles. 
$K(A)$ independent of $A$, then $T$ is a quasiconformal mapping, and vice versa [3]. Therefore the class of all Royden's maps includes the class of all quasiconformal mappings. Furthermore, the inclusion is always proper for every pair of $R_{1}$ and $R_{2}$.

3. A relation to Royden's compactification. The reason we call mappings with property (2) Royden's maps is clarified by the following:

THEOREM 1. A Royden's map $T$ of $R_{1}$ onto $R_{2}$ can be continued to a unique topological mapping $T^{*}$ of $R_{1}^{*}$ onto $R_{2}^{*}$. Conversely, a topological mapping $T^{*}$ of $R_{1}^{*}$ onto $R_{2}^{*}$ always maps $R_{1}$ onto $R_{2}$ and $T=T^{*} \mid R_{1}$ is a Royden's map of $R_{1}$ onto $R_{2}$.

The first assertion is known for quasiconformal mappings [4]. We may summarize Theorem 1 as follows: the topological structure of $R^{*}$ characterizes the quasiconformal structure of $R$ at the ideal boundary.

4. Boundary behavior. The topological extension $T^{*}$ of a Royden's map $T$ of $R_{1}$ onto $R_{2}$ gives a topological mapping of $\Gamma_{1}$ onto $\Gamma_{2}$, where $\Gamma=R^{*}-R$ is the Royden's boundary of $R$. Let $\Delta$ be the Royden's harmonic boundary of $R$, i.e., the totality of regular points in $\Gamma$ with respect to the Dirichlet problem [7]. Then

THEOREM 2. The topological extension $T^{*}$ of a Royden's map $T$ of $R_{1}$ onto $R_{2}$ gives a topological mapping of $\Delta_{1}$ onto $\Delta_{2}$.

The properties $R \in O_{G}, O_{H D}$, or $O_{H D}^{n}$ are all characterized by the set theoretic properties of $\Delta[6]$. Therefore, as a corollary of Theorem 2, we conclude that each of the classes $O_{G}, O_{H D}$, and $O_{H D}^{n}$ is preserved under Royden's maps. This assertion generalizes theorems of Pfluger [8] and Royden [9].

5. The case for the half plane. Let $T$ be a Royden's map of the upper half plane $U=\{z \mid \operatorname{Im}(z)>0\}$ onto itself. We also denote $\partial U=\{z \mid \operatorname{Im}(z)=0\}$ and $\bar{U}=\{z \mid \operatorname{Im}(z) \geqq 0\}$.

TheOREM 3. The map $T$ can be continued to a topological mapping $\bar{T}$ of $\bar{U}$ onto $\bar{U}$.

This is, of course, well known for quasiconformal mappings [1]. Clearly $T$ is both directly and indirectly conformally invariant. Hence we may assume that $\bar{T} \mid \partial U$ is a monotone increasing topological mapping of $(-\infty, \infty)$ onto itself. Then

THEOREM 4. There exists a constant $\rho \geqq 1$ such that 


$$
\rho^{-1} \leqq \frac{\bar{T}(x+t)-\bar{T}(x)}{\bar{T}(x)-\bar{T}(x-t)} \leqq \rho
$$

for any $x \in \partial U$ and $t>0$.

The inequality (3) is often referred to as the $\rho$-condition. The validity of (3) is well known for quasiconformal mappings [2].

From the features of Royden's maps listed above we conclude that Royden's maps are topological mappings which are "quasiconformal at the ideal boundaries."

\section{BIBLIOGRAPHY}

1. L. V. Ahlfors, On quasiconformal mappings, J. Analyse Math. 3 (1953-1954), 1-58, 207-208.

2. A. Beurling and L. V. Ahlfors, The boundary correspondence under quasiconformal mappings, Acta Math. 96 (1956), 125-142.

3. H. Künzi, Quasikonforme Abbildungen, Springer-Verlag, Berlin, 1960.

4. M. Nakai, On a ring isomorphism induced by quasiconformal mappings, Nagoya Math. J. 14 (1959), 201-221.

5. - Algebraic criterion on quasiconformal equivalence of Riemann surfaces, Nagoya Math. J. 16 (1960), 157-184.

6. - A measure on the harmonic boundary of a Riemann surface, Nagoya Math. J. 17 (1960), 181-218.

7. - Genus and classification of Riemann surfaces, Osaka J. Math. 14 (1962), 153-180.

8. A. Pfluger, Sur un propriété de l'application quasi conforme d'une surface de Riemann ouverte, C. R. Acad. Sci. Paris 227 (1948), 25-26.

9. H. L. Royden, A property of quasiconformal mapping, Proc. Amer. Math. Soc. 5 (1954), 266-269.

University of California, Los Angeles and NAgOYa UNIVERSITY 